\title{
Safety, Tolerability, Pharmacokinetics, and Pharmacodynamics of Alirocumab in Healthy Chinese Subjects: A Randomized, Double-Blind, Placebo-Controlled, Ascending Single-Dose Study
}

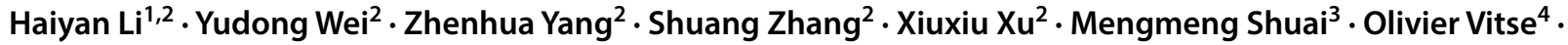 \\ Yiwen $\mathrm{Wu}^{3} \cdot$ Marie T. Baccara-Dinet ${ }^{4} \cdot \mathrm{Yi}_{\mathrm{Z}}$ hang $^{5} \cdot$ Jianyong $\mathrm{Li}^{6}$
}

Published online: 21 February 2020

(c) The Author(s) 2020

\begin{abstract}
Background The addition of alirocumab (a fully human monoclonal antibody to proprotein convertase subtilisin/kexin type 9 [PCSK9]) to background statin therapy provides significant incremental low-density lipoprotein cholesterol (LDL-C) lowering and cardiovascular event risk reduction.

Objectives Our objectives were to assess the safety, tolerability, pharmacokinetics, and pharmacodynamics of single ascending doses of alirocumab in healthy Chinese subjects.

Methods In this double-blind, placebo-controlled, phase I study, 35 Chinese subjects (aged 21-45 years) with baseline LDL-C $>100 \mathrm{mg} / \mathrm{dL}(2.59 \mathrm{mmol} / \mathrm{L})$ were randomized to receive a single $1 \mathrm{~mL}$ subcutaneous injection of alirocumab 75 , 150 , or $300 \mathrm{mg}$, or placebo, and followed up for $\sim 12$ weeks.

Results Treatment-emergent adverse events, most frequently nasal congestion and dry throat, were reported in three of seven or eight subjects in each alirocumab dose group (two of seven in the placebo group). One patient receiving alirocumab $300 \mathrm{mg}$ had a mild local injection-site reaction. No alirocumab recipients demonstrated antidrug antibodies. Maximum alirocumab serum concentrations (6-34 mg/dL) occurred at a median of 3-7 days across the dose groups. Maximum mean LDL-C reductions from baseline were observed on days 8, 15, and 22 with alirocumab 75 (55.3\%), 150 (63.7\%), and $300 \mathrm{mg}$ (73.7\%), respectively. Mean free PCSK9 levels were reduced to below the lower limit of quantification within $4 \mathrm{~h}$ of dosing. Total cholesterol, non-high-density lipoprotein cholesterol, and apolipoprotein B were reduced with alirocumab.

Conclusions In Chinese subjects, alirocumab 75, 150, and $300 \mathrm{mg}$ was safe and well-tolerated. Pharmacokinetic/pharmacodynamic parameters, including clinically meaningful reductions in LDL-C and other lipids/lipoproteins, were consistent with data from Japanese and Western populations.
\end{abstract}

Clinicaltrials.gov identifier: NCT02979015.

Electronic supplementary material The online version of this article (https://doi.org/10.1007/s40256-020-00394-1) contains supplementary material, which is available to authorized users.

Haiyan Li

haiyanli1027@hotmail.com

Extended author information available on the last page of the article 


\section{Key Points}

Data regarding the safety, tolerability, and pharmacokinetic and pharmacodynamic parameters of the proprotein convertase subtilisin/kexin type 9 monoclonal antibody alirocumab in Chinese subjects are limited.

In this double-blind, placebo-controlled, phase I study in 35 healthy Chinese subjects, alirocumab administered as a single ascending subcutaneous dose of 75,150 , or $300 \mathrm{mg}$ was generally safe and well-tolerated.

The pharmacokinetic and pharmacodynamic parameters of alirocumab in healthy Chinese subjects were consistent with those reported for Japanese and Western populations, with pharmacodynamic data showing clinically meaningful reductions in low-density lipoprotein cholesterol and other lipids and lipoproteins. reduction $[11,12]$. Two PCSK9 inhibitors, alirocumab and evolocumab, both fully human monoclonal antibodies to PCSK9, are approved for clinical use in combination with statin/ezetimibe in the USA and Europe [13-15]. Evolocumab is also available in China [16], and alirocumab is currently undergoing regulatory review in this region.

Alirocumab provides significant additional reductions in LDL-C of up to $62 \%$, relative to placebo, when added to background maximally tolerated statin therapy [17], as well as reductions in major adverse cardiovascular events [18]. However, data regarding the safety, pharmacokinetics, and pharmacodynamics of alirocumab in Chinese subjects are limited. For the first time, this study investigated the safety, tolerability, pharmacokinetics, and pharmacodynamics of alirocumab following subcutaneous administration of single ascending doses $(75,150$, or $300 \mathrm{mg})$ in healthy Chinese subjects.

\section{Methods}

\subsection{Study Design and Participants}

This was a phase I, single-center, randomized, doubleblind, placebo-controlled, ascending single-dose study of the safety, tolerability, pharmacokinetics, and pharmacodynamics of alirocumab following subcutaneous administration in healthy Chinese subjects (clinicaltrials.gov identifier: NCT02979015).

The study was conducted at the Peking University Third Hospital, Beijing, China, in accordance with recommendations of the 18th World Health Congress (Helsinki 1964) and all applicable amendments, and the International Conference on Harmonization guidelines for Good Clinical Practice (GCP). The protocol was approved by the relevant independent ethics committees. All participating subjects provided written informed consent.

Healthy male or female subjects (30 planned) aged between 21 and 45 years inclusive, with serum LDL-C > $100 \mathrm{mg} / \mathrm{dL}$ (2.59 mmoL/L) at screening, were eligible for enrollment. Exclusion criteria included a history/ presence of clinically relevant disease (e.g., cardiovascular, pulmonary, hepatic, or renal) or signs of acute illness (for additional eligibility/exclusion criteria, see Supplementary Table 1).

The study comprised a screening period from 2 to 28 days prior to inclusion (days - 28 to -2), a 5-day in-house period including 1 treatment day (days -1 to 4 , with treatment on day 1), clinic visit days \pm 2 days (days 8, 11, 15, 22, 29, 43, and 64), and an end of study (EOS) visit (day $85 \pm 2$ days). The total study duration for each subject, not including the screening period, was $\sim 12$ weeks; duration of follow-up was up to 113 days, including screening. 
Subjects were randomized to one of three sequential ascending dose groups (each completed before initiating the next dose group) with ten subjects planned per group, eight to receive alirocumab and two to receive matching placebo: group 1, a single dose of alirocumab $75 \mathrm{mg}(75 \mathrm{mg} /$ $\mathrm{mL} \times 1 \mathrm{~mL}$ ) or placebo; group 2, a single dose of alirocumab $150 \mathrm{mg}(150 \mathrm{mg} / \mathrm{mL} \times 1 \mathrm{~mL})$ or placebo; and group 3 , a single dose of alirocumab $300 \mathrm{mg}(2 \times 150 \mathrm{mg} / \mathrm{mL} \times 1 \mathrm{~mL})$ or placebo. Each treatment was administered subcutaneously via prefilled pen in the abdomen on day 1 under fasting conditions. At the time of the discussion of the study design (16 October 2012), 75 and $150 \mathrm{mg}$ were the therapeutic doses of alirocumab used in the worldwide phase III program (75 mg every 2 weeks [Q2W]/up to $150 \mathrm{mg} \mathrm{Q2W).} \mathrm{The} \mathrm{highest}$ dose of $300 \mathrm{mg}$ was well-tolerated in previous studies [19, 20]; 75,150 , and $300 \mathrm{mg}$ were therefore selected for this study to provide relevant data regarding the safety margin and dose proportionality. By the time of study initiation (29 November 2016), alirocumab $300 \mathrm{mg}$ every 4 weeks had been investigated in the phase III program [21].

\subsection{Safety Assessments}

Safety was assessed by physical examination and monitoring of clinical laboratory and electrocardiogram (ECG) parameters, vital signs, and adverse events (AEs) spontaneously reported by the subject or observed by the investigator, including local tolerability/sensitivity reactions (further details are provided in the electronic supplementary material). Local tolerability parameters monitored included injection-site pain. Erythema or edema at the injection site were assessed qualitatively as mild, moderate, or severe.

AEs were coded according to the Medical Dictionary for Regulatory Activities (MedDRA, version 20.1). Treatment-emergent AEs (TEAEs) were defined as those that occurred, worsened, or became serious during the period from alirocumab or placebo injection (day 1) until EOS (day $85 \pm 2$ ). A serious AE (SAE) was considered as any untoward medical occurrence/important event that at any dose resulted in death, was life threatening, required hospitalization, or resulted in significant disability/incapacity. TEAEs were listed by treatment group, primary system organ class (MedDRA order), and preferred term.

\subsection{Pharmacokinetic and Antidrug Antibody Assessments}

Blood samples for determination of alirocumab serum concentrations were collected before study drug administration and on days 1 (at 4 and $8 \mathrm{~h}$ ), 2 (at $24 \mathrm{~h}$ ), 3 (48 h), 4 (72 h), 8 (168 h), 11 (240 h), 15 (336 h), 22 (504 h), 29 (672 h), 43 (1008 h), 64 (1512 h), and 85 (2016 h) post-dose.
Total concentrations of alirocumab (free alirocumab and in PCSK9:alirocumab complexes) were determined in acid-treated human serum using a validated enzymelinked immunosorbent assay (ELISA), with a lower limit of quantification (LLOQ) of $78 \mathrm{ng} / \mathrm{mL}$ (Regeneron Pharmaceuticals, Inc., Tarrytown, NY, USA) [22]. Free and total PCSK9 levels were determined by validated ELISA assay (Regeneron Pharmaceuticals, Inc.) [22]. The lower limits of detection for total and free PCSK9 were $156 \mathrm{ng} / \mathrm{mL}$ and $31.2 \mathrm{ng} / \mathrm{mL}$, respectively. Antidrug antibodies (ADAs) to alirocumab were analyzed by the Regeneron Clinical Bioanalysis group using a validated, nonquantitative, titer-based bridging immunoassay [23].

Alirocumab serum pharmacokinetic parameters were calculated using noncompartmental analysis with validated software (pharmacokinetic data management system version 3 with Phoenix version 1.4). Calculated alirocumab pharmacokinetic parameters included maximum serum concentration $\left(C_{\max }\right)$; time to reach $C_{\max }\left(t_{\max }\right)$; area under the serum concentration versus time curve (AUC) extrapolated from time zero to infinity $\left(\mathrm{AUC}_{\infty}\right.$ ) $\mathrm{AUC}$ from time zero to day $15\left(\mathrm{AUC}_{0-\mathrm{D} 14}\right)$; $\mathrm{AUC}$ from time zero to day $29\left(\mathrm{AUC}_{0-\mathrm{D} 28}\right)$; AUC from time zero to time of last measurable concentration $\left(\mathrm{AUC}_{\text {last }}\right)$; the last concentration above the limit of quantification $\left(C_{\text {last }}\right)$; time to $C_{\text {last }}$; apparent total body clearance $(\mathrm{CL} / F)$ from plasma; mean residence time; terminal elimination half-life $\left(t_{1 / 2 z}\right.$; alirocumab is a protein that is degraded to small peptides and amino acids so has a concentrationdependent clearance); and apparent distribution volume at steady state $\left(V_{\mathrm{ss}} / F\right)$.

\subsection{Pharmacodynamic Assessments}

Blood sampling for lipid/lipoprotein parameters, LDL-C, total cholesterol (TC), high-density lipoprotein (HDL-C), triglycerides, apolipoprotein (Apo) B, ApoA1, and lipoprotein a $[\mathrm{Lp}(\mathrm{a})]$ were performed in the morning under conditions of fasting ( $\geq 10 \mathrm{~h}$ ) and no smoking (for further detail, see the electronic supplementary material).

The primary pharmacodynamic variables assessed were percent and absolute change from baseline in calculated LDL-C at each visit. LDL-C was calculated using the Friedewald formula [24]. Secondary pharmacodynamic variables assessed were percent and absolute change from baseline in TC, HDL-C, triglycerides, non-HDL-C, very lowdensity lipoprotein cholesterol (VLDL-C), ApoB, ApoA1, and $L p(a)$ at each visit.

\subsection{Statistical Analysis}

The sample size for this study was calculated based upon empirical considerations. We estimated that allocation of six subjects in the placebo groups and 24 in the active dose 
groups would provide at least $90 \%$ power to detect a treatment difference of $30 \%$ (standard deviation $15 \%$ ) versus placebo for percentage change from baseline in LDL-C, with a $5 \%$ significance level in a two-sided $t$ test. There was no adjustment for multiplicity due to multiple comparisons.

The safety population included all randomized subjects exposed to study treatment, regardless of the amount of treatment administered. Safety data were analyzed by review of descriptive statistics and individual data for AEs, clinical laboratory values, vital signs, and ECG parameters. The immunogenicity of alirocumab was assessed by summarizing the number and percentage of subjects who tested positive or negative for ADAs. The pharmacokinetic population included all subjects with no important deviations related to alirocumab administration and who were considered to have sufficient and interpretable pharmacokinetic data; subjects who received only placebo were not included in this population. Alirocumab, free PCSK9, and total PCSK9 concentrations during the treatment period were summarized by treatment group and visit using descriptive statistics. No formal statistical comparisons were performed, and pharmacokinetic data are summarized descriptively. All subjects with no important deviations affecting pharmacodynamic evaluation, for whom some pharmacodynamic data were considered available, were included in the pharmacodynamic population.

\section{Results}

\subsection{Baseline Subject Characteristics}

Initially, 31 healthy subjects were randomized in the study. Four subjects (two each in the alirocumab $150 \mathrm{mg}$ and alirocumab $300 \mathrm{mg}$ groups) were noncompliant with GCP as they had used others' identification cards and were excluded from any of the analyses; these subjects were replaced. Overall, 31 subjects were randomized, treated, and analyzed: eight subjects in each of the alirocumab 75, 150, and $300 \mathrm{mg}$ groups and seven subjects in the placebo group. One placebo recipient did not finish the study procedure because they experienced an SAE. The first subject was enrolled on 29 November 2016, and 31 subjects had completed the study treatment period by 27 November 2017. Figure 1 shows the flow of subjects through the study.

Baseline characteristics, including mean body mass index, were generally similar between the alirocumab and placebo groups (Table 1), except that the placebo group contained fewer males (42.9 vs. 83.3\% across the alirocumab groups). Mean baseline LDL-C (111.8 mg/dL [2.89 mmol/L]) for all groups was similar between the alirocumab 75 (111.4 mg/ $\mathrm{dL}$ [2.88 mmol/L]), 150 (109.8 $\mathrm{mg} / \mathrm{dL}$ [2.84 mmol/L]), and $300 \mathrm{mg}(111.8 \mathrm{mg} / \mathrm{dL}[2.89 \mathrm{mmol} / \mathrm{L}])$ groups and placebo (114.8 mg/dL [2.97 mmol/L]).

Although none of the subjects took previous medications, some did take concomitant medications in response to AEs: one subject in the placebo group, two in the alirocumab $75 \mathrm{mg}$ group, and three in the alirocumab $150 \mathrm{mg}$ group.

\subsection{Safety}

Alirocumab was generally well-tolerated in the 31 healthy Chinese subjects included in the safety population. Overall, TEAEs were reported by three of eight, six of eight, and seven of eight subjects in the alirocumab 75, 150, and $300 \mathrm{mg}$ groups, respectively, compared with two of seven in the placebo groups (Table 2). No single TEAE showed a clear dose-response relationship. No alirocumab recipients reported severe TEAEs or treatment-emergent SAEs. No deaths were reported during the study. One subject in the

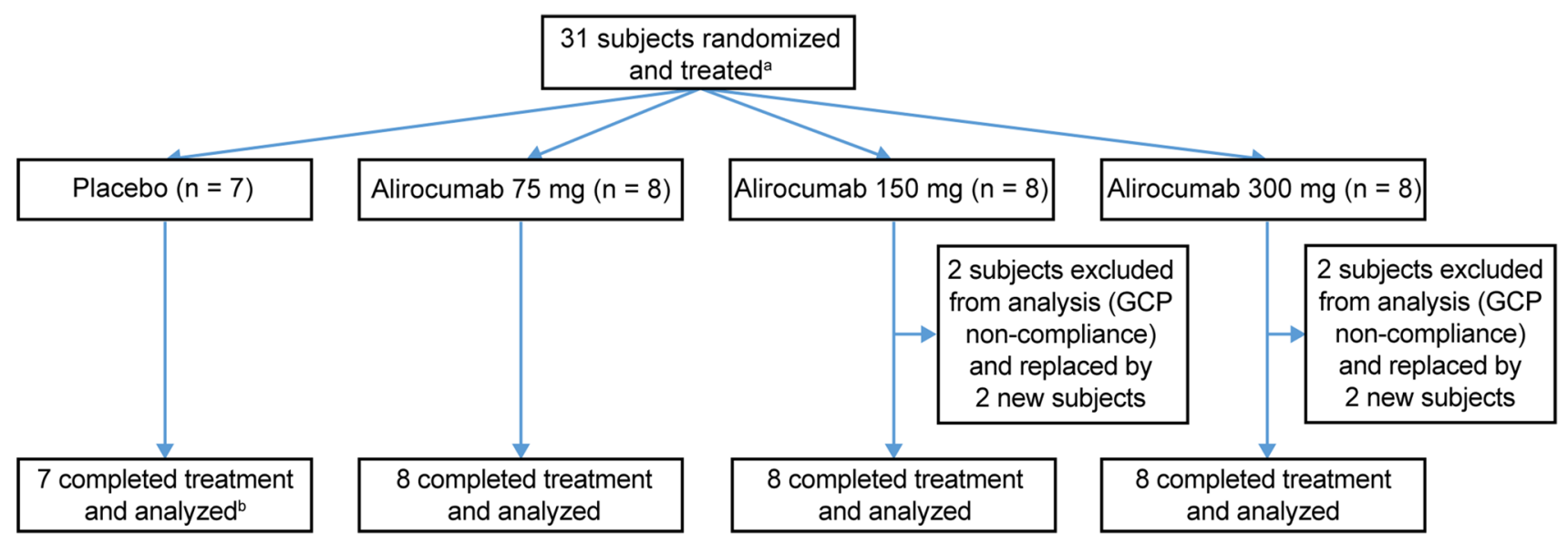

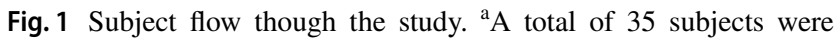
enrolled: 31 were initially randomized, but four subjects were replaced because of non-compliance. ${ }^{b}$ One treated subject did not fin- ish the study visits because of a serious adverse event (acute appendicitis). GCP good clinical practice 
Table 1 Baseline characteristics of healthy Chinese subjects

\begin{tabular}{llllll}
\hline Characteristics & \multicolumn{2}{l}{ Alirocumab } & & \multirow{2}{*}{ Placebo $(n=7)$} & \\
\cline { 2 - 4 } & $75 \mathrm{mg}(n=8)$ & $150 \mathrm{mg}(n=8)$ & $300 \mathrm{mg}(n=8)$ & & $36.0 \pm 8.9$ \\
\hline Age, years & $30.3 \pm 5.7$ & $36.5 \pm 5.8$ & $36.0 \pm 7.3$ & $3(42.9)$ & $23(74.2)$ \\
Male & $8(100.0)$ & $7(87.5)$ & $5(62.5)$ & $62.3 \pm 6.7$ & $69.1 \pm 9.9$ \\
Weight, $\mathrm{kg}$ & $73.5 \pm 11.9$ & $74.2 \pm 6.9$ & $65.3 \pm 8.9$ & $1.57 \pm 0.08$ & $1.66 \pm 0.09$ \\
Height, $\mathrm{m}$ & $1.73 \pm 0.06$ & $1.71 \pm 0.08$ & $1.63 \pm 0.04$ & $25.4 \pm 1.7$ & $25.0 \pm 2.4$ \\
BMI, $\mathrm{kg} / \mathrm{m}^{2}$ & $24.49 \pm 2.9$ & $25.6 \pm 2.3$ & $24.6 \pm 2.8$ & $512.6 \pm 124.1$ & $423.1 \pm 104.5$ \\
Total PCSK9, ng/mL & $353.3 \pm 76.2$ & $402.3 \pm 102.4$ & $435.5 \pm 55.2$ & $199.9 \pm 48.2$ & $161.6 \pm 47.0$ \\
Free PCSK9, $\mathrm{ng} / \mathrm{mL}$ & $139.8 \pm 38.9$ & $149.9 \pm 52.2$ & $161.8 \pm 32.8$ & $114.8 \pm 22.8$ & $111.8 \pm 22.4$ \\
LDL-C, $\mathrm{mg} / \mathrm{dL}$ & $111.4 \pm 22.0$ & $109.8 \pm 29.0$ & $111.8 \pm 19.3$ & $2.97 \pm 0.59$ & $2.89 \pm 0.58$ \\
LDL-C, $\mathrm{mmol} / \mathrm{L}$ & $2.88 \pm 0.57$ & $2.84 \pm 0.75$ & $2.89 \pm 0.50$ & & \\
\hline
\end{tabular}

Data are presented as mean \pm standard deviation or $n(\%)$ unless otherwise indicated

$B M I$ body mass index, $L D L-C$ low-density lipoprotein cholesterol, PCSK 9 proprotein convertase subtilisin/kexin type 9

placebo group (14.3\%) reported an SAE (acute appendicitis) during study treatment, which was not considered related to the study medication by the investigator. All TEAEs reported by subjects in the alirocumab groups were of mild or moderate intensity and resolved without sequelae.

The most frequently reported TEAEs by system organ class in the alirocumab and placebo groups were "infections and infestations," "respiratory, thoracic, and mediastinal disorders," and "gastrointestinal disorders" (Table 2), which were reported by a similar proportion of subjects in each group. Nasal congestion (three with alirocumab $300 \mathrm{mg}$ and one with placebo) and dry throat (two with alirocumab $300 \mathrm{mg}$ ) were the most frequently reported TEAEs classified by preferred term. One subject receiving alirocumab $300 \mathrm{mg}$ experienced a mild local injection-site reaction (erythema and itching on the right side of the injection site) on the day after study drug administration, which resolved spontaneously within 2 days.

There were no notable changes from baseline in vital signs, laboratory values, or ECG parameters, and no subjects showed potentially clinically significant abnormalities (PCSA) for liver or renal function parameters. Notably, no subjects had alanine transaminase or aspartate transaminase values $>3 \times$ the upper limit of normal (ULN), alkaline phosphatase values $>1.5 \times \mathrm{ULN}$, or total bilirubin values $>1.5 \times$ ULN. Two subjects receiving alirocumab $150 \mathrm{mg}$ had on-treatment PCSAs for biochemistry parameters. One subject with a baseline glucose of $5.5 \mathrm{mmol} / \mathrm{L}$ had a fasting glucose level above the ULN $(7.2 \mathrm{mmol} / \mathrm{L})$ on day 43 , which returned to normal by the EOS visit. Another subject with baseline creatine phosphokinase $(\mathrm{CPK})$ of $1.36 \times \mathrm{ULN}$ had a transient elevation to more than $3 \times$ ULN on day 4 . CPK levels remained elevated at day $7(1.72 \times \mathrm{ULN})$ and day $10(1.48 \times \mathrm{ULN})$, with a return to $<3 \times$ ULN on day 15 $(0.63 \times \mathrm{ULN})$.

\subsubsection{Immunogenicity}

Across the three alirocumab dose groups, all subjects were ADA negative. A low ADA positive titer (60) was reported for one subject in the placebo group on a single occasion on day 85 .

\subsection{Pharmacokinetic Analysis}

All subjects randomized to alirocumab 75, 150, and $300 \mathrm{mg}$ were included in the pharmacokinetic population $(n=24)$. Each of these subjects demonstrated measurable alirocumab levels; by contrast, no placebo recipients had detectable serum alirocumab levels. Figure 2 shows the mean serum alirocumab concentration versus time after single subcutaneous administration of alirocumab 75,150 , or $300 \mathrm{mg}$. $C_{\max }$ 6-34 mg/L was observed at a median of 3-7 days across the three dose groups, and $\mathrm{AUC}_{\text {last }}$ and $\mathrm{AUC}_{\infty}$ were similar within each dose group (Table 3 ). The total variability (coefficient of variation) of $C_{\text {max }}, \mathrm{AUC}_{\text {last }}$, and $\mathrm{AUC}_{\infty}$ ranged from 30.0 to $53.5 \%$, from 27.9 to $45.3 \%$, and from 26.5 to $44.8 \%$, respectively. Apparent CL/F decreased from $0.9 \mathrm{~L} /$ day following single subcutaneous administration of alirocumab 75 and $150 \mathrm{mg}$ to $0.5 \mathrm{~L} /$ day with $300 \mathrm{mg}$; respective values for $V_{s \mathrm{~s}} / F$ were 10,12 , and $7 \mathrm{~L}$, respectively.

\subsubsection{Dose Proportionality}

Comparison of the main pharmacokinetic parameters obtained with the lowest $(75 \mathrm{mg})$ and highest $(300 \mathrm{mg}$ ) doses showed that alirocumab exposure increased slightly more than expected from dose proportionality. In statistical assessment of dose proportionality (analysis of variance model estimated with $90 \%$ confidence interval $[\mathrm{CI}]$ for pairwise dose increases), the 4.00 -fold increase in dose from 75 to 
Table 2 Treatment-emergent adverse events

\begin{tabular}{|c|c|c|c|c|}
\hline \multirow[t]{2}{*}{ TEAE $^{\mathrm{a}}$} & \multicolumn{3}{|l|}{ Alirocumab } & \multirow[t]{2}{*}{ Placebo $(n=7)$} \\
\hline & $75 \mathrm{mg}(n=8)$ & $150 \mathrm{mg}(n=8)$ & $300 \mathrm{mg}(n=8)$ & \\
\hline TEAEs & $3(37.5)$ & $6(75.0)$ & $7(87.5)$ & $2(28.6)$ \\
\hline Treatment-emergent SAEs & 0 & 0 & 0 & $1(14.3)^{\mathrm{b}}$ \\
\hline \multicolumn{5}{|l|}{ TEAEs by system organ class, preferred term } \\
\hline Any class & $3(37.5)$ & $6(75.0)$ & $7(87.5)$ & $2(28.6)$ \\
\hline Infections and infestations & $1(12.5)$ & $2(25.0)$ & $1(12.5)$ & $2(28.6)$ \\
\hline Folliculitis & 0 & 0 & $1(12.5)$ & 0 \\
\hline Appendicitis & 0 & 0 & 0 & $1(14.3)$ \\
\hline Tonsillitis & $1(12.5)$ & $1(12.5)$ & 0 & 0 \\
\hline Upper respiratory tract infection & 0 & $1(12.5)$ & 0 & $1(14.3)$ \\
\hline Psychiatric disorders & $1(12.5)$ & 0 & 0 & 0 \\
\hline Insomnia & $1(12.5)$ & 0 & 0 & 0 \\
\hline Respiratory, thoracic, and mediastinal disorders & 0 & $2(25.0)$ & $3(37.5)$ & $1(14.3)$ \\
\hline Nasal congestion & 0 & 0 & $3(37.5)$ & $1(14.3)$ \\
\hline Dry throat & 0 & 0 & $2(25.0)$ & 0 \\
\hline Oropharyngeal pain & 0 & $1(12.5)$ & $1(12.5)$ & $1(14.3)$ \\
\hline Rhinorrhea & 0 & $1(12.5)$ & 0 & 0 \\
\hline Gastrointestinal disorders & $2(25.0)$ & $2(25.0)$ & $1(12.5)$ & $1(14.3)$ \\
\hline Abdominal pain & $1(12.5)$ & 0 & $1(12.5)$ & $1(14.3)$ \\
\hline Diarrhea & $1(12.5)$ & $1(12.5)$ & $1(12.5)$ & 0 \\
\hline Enteritis & 0 & $1(12.5)$ & 0 & 0 \\
\hline Mouth ulceration & $1(12.5)$ & 0 & 0 & 0 \\
\hline Toothache & 0 & $1(12.5)$ & 0 & 0 \\
\hline Hepatobiliary disorders & 0 & 0 & 0 & $1(14.3)$ \\
\hline Hepatic steatosis & 0 & 0 & 0 & $1(14.3)$ \\
\hline Skin and subcutaneous tissue disorders & 0 & $3(37.5)$ & $2(25.0)$ & 0 \\
\hline Pruritus & 0 & $1(12.5)$ & $1(12.5)$ & 0 \\
\hline Rash & 0 & $1(12.5)$ & $1(12.5)$ & 0 \\
\hline Skin exfoliation & 0 & $1(12.5)$ & 0 & 0 \\
\hline Musculoskeletal and connective tissue disorders & 0 & 0 & $1(12.5)$ & $1(14.3)$ \\
\hline Back pain & 0 & 0 & $1(12.5)$ & 0 \\
\hline Pain in extremity & 0 & 0 & 0 & $1(14.3)$ \\
\hline General disorders and administration-site conditions & 0 & 0 & $2(25.0)$ & 0 \\
\hline Asthenia & 0 & 0 & $1(12.5)$ & 0 \\
\hline Injection-site reaction & 0 & 0 & $1(12.5)$ & 0 \\
\hline Investigations & 0 & $1(12.5)$ & 0 & 0 \\
\hline Blood creatine phosphokinase increased & 0 & $1(12.5)$ & 0 & 0 \\
\hline Injury, poisoning, and procedural complications & 0 & 0 & $1(12.5)$ & 0 \\
\hline Ligament sprain & 0 & 0 & $1(12.5)$ & 0 \\
\hline
\end{tabular}

Data are presented as $n(\%)$ unless otherwise indicated

$S A E$ serious adverse event, TEAE treatment-emergent adverse event

${ }^{a}$ No TEAEs led to treatment discontinuation in any group as this was a single-dose study

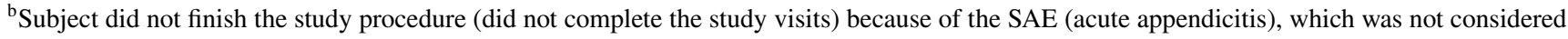
related to the investigational medicinal product by the investigator; the subject had a medical history of chronic appendicitis

$300 \mathrm{mg}$ resulted in a 5.38-fold (90\% CI 3.84-7.55) increase in $C_{\max }$, an 8.32-fold (90\% CI 6.12-11.32) increase in AUC last, and an 8.00-fold (90\% CI 5.92-10.81) increase in AUC $\infty$. When comparing the exposure of the 75 and $150 \mathrm{mg}$ dose groups, the point estimates for $C_{\max }, \mathrm{AUC}_{\text {last }}$, and $\mathrm{AUC}_{\infty}$ were closer to an expected twofold increase $(1.80,2.17$, and 2.10 , respectively); for comparison of the 150 and $300 \mathrm{mg}$ 
Fig. 2 Mean serum alirocumab concentration versus time profiles after single subcutaneous administration of alirocumab 75,150 , or $300 \mathrm{mg}$ (linear scale). $L O Q$ limit of quantification, $S D$ standard deviation

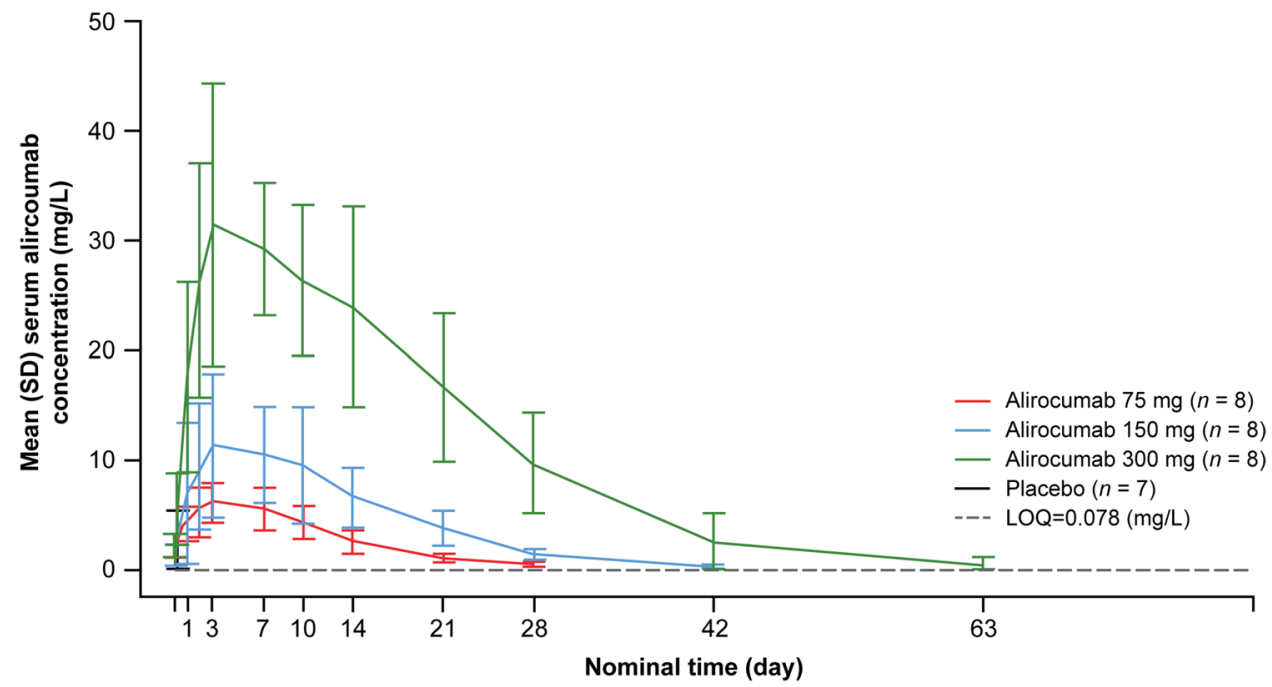

Table 3 Pharmacokinetic parameters following subcutaneous administration of alirocumab 75, 150, or 300 mg in healthy Chinese subjects

\begin{tabular}{|c|c|c|c|}
\hline \multirow[t]{2}{*}{ Parameter } & \multicolumn{3}{|l|}{ Alirocumab } \\
\hline & $75 \mathrm{mg}(n=8)$ & $150 \mathrm{mg}(n=8)$ & $300 \mathrm{mg}(n=8)$ \\
\hline$C_{\max }, \mathrm{mg} / \mathrm{L}$ & $\begin{array}{l}6.32 \pm 1.90 \\
(6.07)[30.0]\end{array}$ & $\begin{array}{l}12.3 \pm 6.60 \\
(10.9)[53.5]\end{array}$ & $\begin{array}{l}34.0 \pm 10.6 \\
(32.7)[31.2]\end{array}$ \\
\hline$t_{\max }$, days $^{\mathrm{a}}$ & $3.00(2.00-7.01)$ & $5.00(2.00-9.98)$ & $6.99(3.00-10.00)$ \\
\hline $\mathrm{AUC}_{0-\mathrm{D} 14}, \mathrm{mg} \cdot \mathrm{day} / \mathrm{L}$ & $\begin{array}{l}64.4 \pm 20.2 \\
(61.8)[31.4]\end{array}$ & $\begin{array}{l}126.0 \pm 65.0 \\
(114)[51.4]\end{array}$ & $\begin{array}{l}365.0 \pm 106.0 \\
(353)[28.9]\end{array}$ \\
\hline $\mathrm{AUC}_{0-\mathrm{D} 28}, \mathrm{mg} \cdot \mathrm{day} / \mathrm{L}$ & $\begin{array}{l}83.2 \pm 24.0 \\
(80.3)[28.9]\end{array}$ & $\begin{array}{l}181.0 \pm 85.1 \\
(165)[47.1]\end{array}$ & $\begin{array}{l}598.0 \pm 195.0 \\
(574)[32.6]\end{array}$ \\
\hline $\mathrm{AUC}_{\text {last }}, \mathrm{mg} \cdot \mathrm{day} / \mathrm{L}$ & $\begin{array}{l}84.2 \pm 23.5 \\
(81.4)[27.9]\end{array}$ & $\begin{array}{l}192.0 \pm 87.1 \\
(177)[45.3]\end{array}$ & $\begin{array}{l}717.0 \pm 288.0 \\
(678)[40.2]\end{array}$ \\
\hline $\mathrm{AUC}_{\infty}, \mathrm{mg} \cdot \mathrm{day} / \mathrm{L}$ & $\begin{array}{l}87.6 \pm 23.2 \\
(85.0)[26.5]\end{array}$ & $\begin{array}{l}194.0 \pm 86.8 \\
(179)[44.8]\end{array}$ & $\begin{array}{l}719.0 \pm 288 \\
(680)[40.0]\end{array}$ \\
\hline$C_{\text {last }}, \mathrm{mg} / \mathrm{dL}$ & $\begin{array}{l}0.379 \pm 0.255 \\
(0.284)[67.3]\end{array}$ & $\begin{array}{l}0.235 \pm 0.066 \\
(0.266)[27.9]\end{array}$ & $\begin{array}{l}0.258 \pm 0.215 \\
(0.206)[83.2]\end{array}$ \\
\hline$t_{\text {last }}$, days $^{\mathrm{a}}$ & $28.06(28.00-43.06)$ & $42.02(41.97-42.11)$ & $63.00(42.03-84.02)$ \\
\hline$t_{1 / 2 z \text {, days }}$ & $\begin{array}{l}6.07 \pm 1.55 \\
(5.88)[25.5]\end{array}$ & $\begin{array}{l}5.51 \pm 0.789 \\
(5.46)[14.3]\end{array}$ & $\begin{array}{l}6.58 \pm 1.22 \\
(6.50)[18.5]\end{array}$ \\
\hline$V_{\mathrm{ss}} / F, \mathrm{~L}$ & $\begin{array}{l}9.78 \pm 3.37 \\
(9.27)[34.4]\end{array}$ & $\begin{array}{l}11.5 \pm 5.86 \\
(10.3)[50.8]\end{array}$ & $\begin{array}{l}6.96 \pm 1.75 \\
(6.75)[25.1]\end{array}$ \\
\hline MRT, days & $\begin{array}{l}10.6 \pm 1.76 \\
(10.5)[16.5]\end{array}$ & $\begin{array}{l}12.3 \pm 1.55 \\
(12.3)[12.5]\end{array}$ & $\begin{array}{l}15.4 \pm 2.19 \\
(15.3)[14.2]\end{array}$ \\
\hline $\mathrm{CL} / F, \mathrm{~L} /$ day & $\begin{array}{l}0.908 \pm 0.224 \\
(0.882)[24.7]\end{array}$ & $\begin{array}{l}0.908 \pm 0.378 \\
(0.840)[41.6]\end{array}$ & $\begin{array}{l}0.461 \pm 0.134 \\
(0.441)[29.1]\end{array}$ \\
\hline
\end{tabular}

Data are presented as mean \pm standard deviation (geometric mean) [coefficient of variation \%] unless otherwise specified

$A U C$ area under the serum concentration versus time curve, $A U C_{0-D 14}$ AUC from time zero to day $15, A U C_{0-D 28}$ AUC from time zero to day 29 , $A U C_{\infty}$ AUC from time zero to infinity, $A U C_{\text {last }}$ AUC from time zero to time of last measurable concentration, $C_{\text {last }}$ last concentration above the limit of quantification, $C L / F$ clearance relative to bioavailability (dose/AUC), $C_{\max }$ maximum serum concentration, $M R T$ mean residence time, $t_{1 / 2 \text {, }}$, terminal elimination half-life, $t_{\text {last }}$ time to $C_{\text {last }}, t_{\max }$ time to reach $C_{\max }, V_{s s} / F$ distribution volume at steady state $(\mathrm{CL} / F \times \mathrm{MRT})$

${ }^{\mathrm{a}}$ Median (min-max)

dose groups, the point estimates were 3.00, 3.84, and 3.81, respectively.

\subsection{Pharmacodynamic Analysis}

\subsubsection{LDL-C Lowering}


Fig. 3 Mean a percentage and $\mathbf{b}$ absolute change from baseline in LDL-C to EOS after singledose alirocumab or placebo administration. Baseline $=$ day 1 pre-dose assessment. EOS end of study, $L D L-C$ low-density lipoprotein cholesterol, $S D$ standard deviation
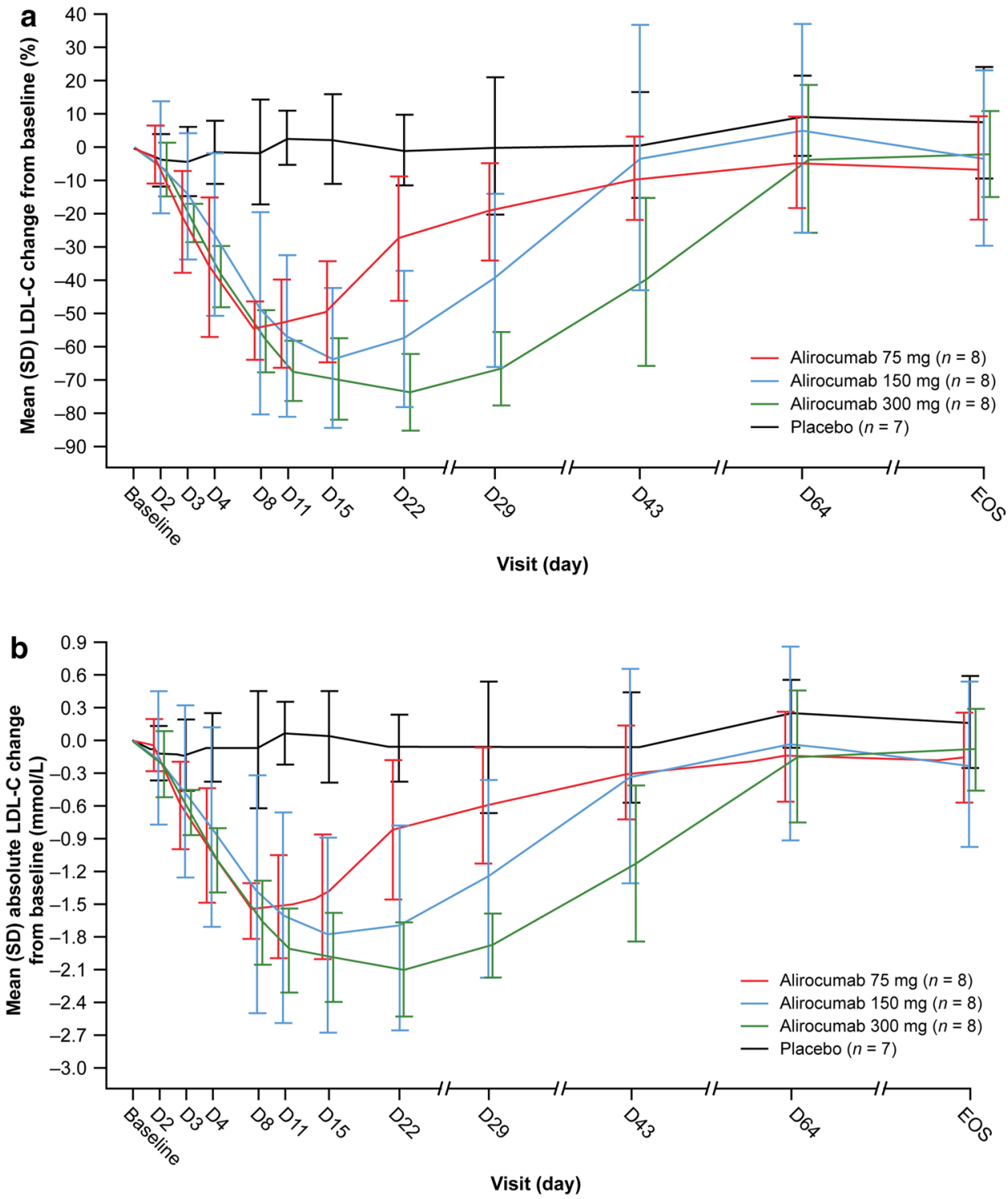

Among the three alirocumab groups, the first observed reduction in LDL-C relative to placebo with alirocumab 75,150 , and $300 \mathrm{mg}$ was on days 3 , 4, and 3, respectively, whereas the maximum mean reduction in LDL-C was observed on days 8 (55.3\%), 15 (63.7\%), and 22 (73.7\%), respectively (Fig. 3a). The reduction in LDL-C with alirocumab 75, 150, and $300 \mathrm{mg}$ was maintained until days 15 , 29 , and 43, respectively. By contrast, in the placebo group, LDL-C levels remained relatively unchanged from baseline to day 64 and EOS.

The maximum absolute changes in LDL-C with alirocumab 75,150 , and $300 \mathrm{mg}$ were $-60.8(-1.57),-69.6$ $(-1.80)$, and $-81.8 \mathrm{mg} / \mathrm{dL}(-2.11 \mathrm{mmol} / \mathrm{L})$, respectively (Fig. 3b). Overall, the magnitude and duration of LDL-C reductions were positively associated with the alirocumab dose administered.

\subsubsection{Free and Total PCSK9 Concentration}

Figure 4a shows the mean free and total PCSK9 concentrations in serum versus time profiles after single subcutaneous administration of alirocumab 75, 150, and $300 \mathrm{mg}$. Mean baseline free PCSK 9 concentrations were similar across the 75,150 , and $300 \mathrm{mg}$ dose groups $(140,150$, and $162 \mathrm{ng} / \mathrm{mL}$, respectively). Mean free PCSK9 concentrations decreased below the LLOQ within $4 \mathrm{~h}$ post-dose and remained below the LLOQ for 10, 14, and 28 days after subcutaneous administration of alirocumab 75, 150, and $300 \mathrm{mg}$, respectively; these returned to baseline values on days 28,42 , and 63 , respectively.

Prior to single subcutaneous administration of alirocumab 75,150 , and $300 \mathrm{mg}$ on day 1 , mean total PCSK9 levels were similar across the groups $(353,402$, and $435 \mathrm{ng} / \mathrm{mL}$, respectively). After alirocumab single-dose administration, mean 
Fig. 4 Mean $\mathbf{a}$ free and $\mathbf{b}$ total PCSK9 concentrations in serum over time after single subcutaneous administration of alirocumab 75,150 , or $300 \mathrm{mg}$. Baseline $=$ day 1 pre-dose assessment. PCSK 9 proprotein convertase subtilisin/kexin type 9, SD standard deviation
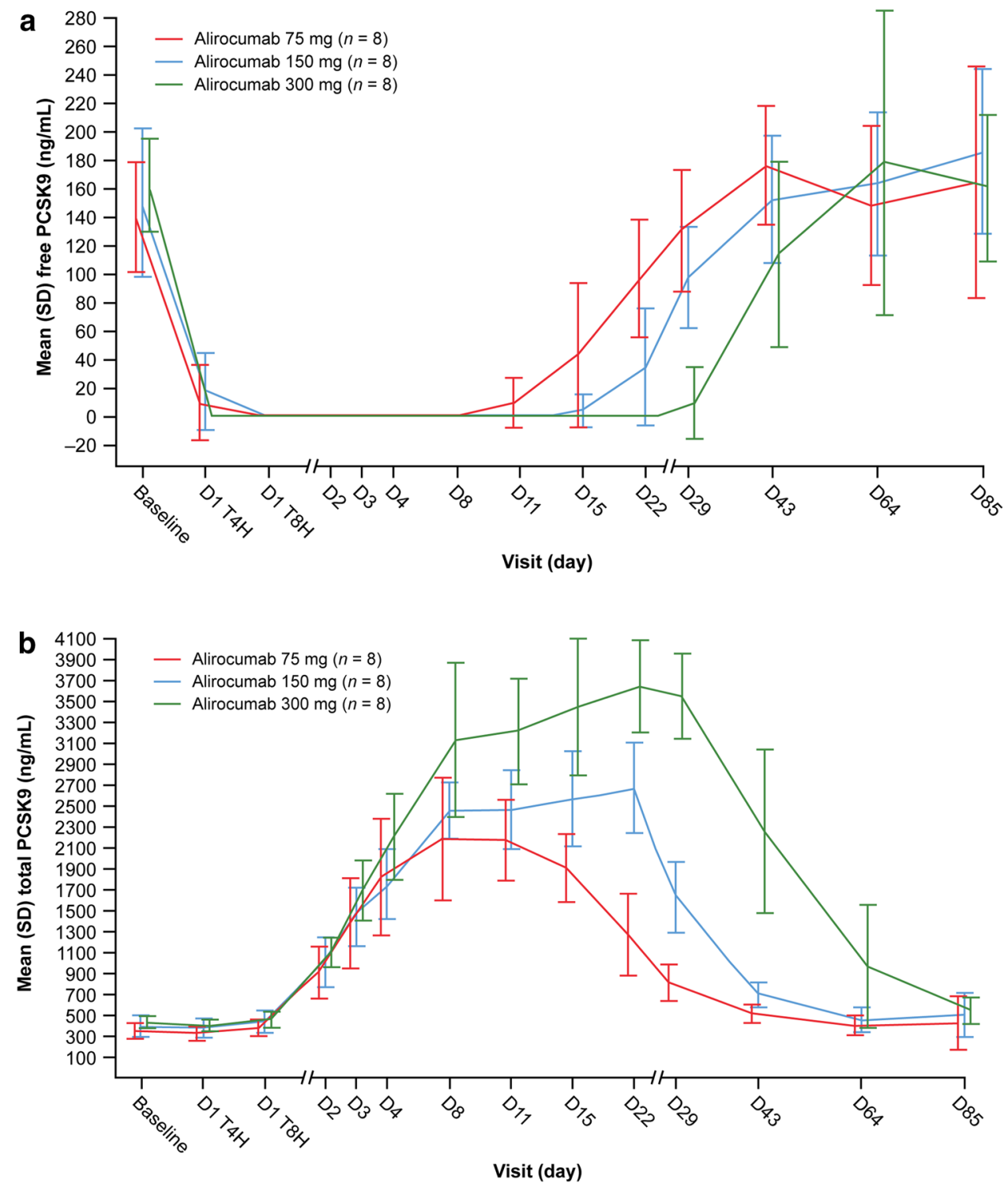

total PCSK9 concentrations increased from days 3 to 7 and reached a plateau that was maintained to days 14,24 , and 28 with alirocumab 75, 150, and $300 \mathrm{mg}$, respectively; plateau levels were higher in the higher dose groups (Fig. 4b). Mean total PCSK9 levels returned to baseline values from days 42 $(75 \mathrm{mg})$ to $84(300 \mathrm{mg})$.

\subsubsection{Relationship Between Alirocumab, PCSK9, and LDL-C Levels}

Figure 5 shows the relationships between alirocumab, free PCSK9, and percentage change in LDL-C levels over time following subcutaneous administration of alirocumab 75 , 150 , and $300 \mathrm{mg}$.

\subsubsection{Other Lipid/Lipoprotein Lowering}

In the three alirocumab groups, reductions were also observed in levels of TC, non-HDL-C, and ApoB, with the maximum reductions seen between days 8 and 22 (Fig. 6). Although there were no significant changes in HDL-C, triglycerides, VLDL-C, ApoA1, and Lp(a), trends were observed for increases in HDL-C and ApoA1 and decreases in triglycerides, VLDL-C, and Lp(a); these lipid/lipoprotein parameters remained unchanged in the placebo group.

\section{Discussion}

In this first study of alirocumab in healthy Chinese subjects with LDL-C $>100 \mathrm{mg} / \mathrm{dL}(2.59 \mathrm{mmol} / \mathrm{L})$, subcutaneous administration of single alirocumab doses $(75,150$, 
Fig. 5 Relationship between alirocumab, free PCSK9, and mean percentage change in LDL-C levels following singledose subcutaneous administration of alirocumab a 75, b 150, and $\mathbf{c} 300 \mathrm{mg}$. Baseline = day 1 pre-dose assessment. $L D L-C$ low-density lipoprotein cholesterol, $P C S K 9$ proprotein convertase subtilisin/kexin type 9
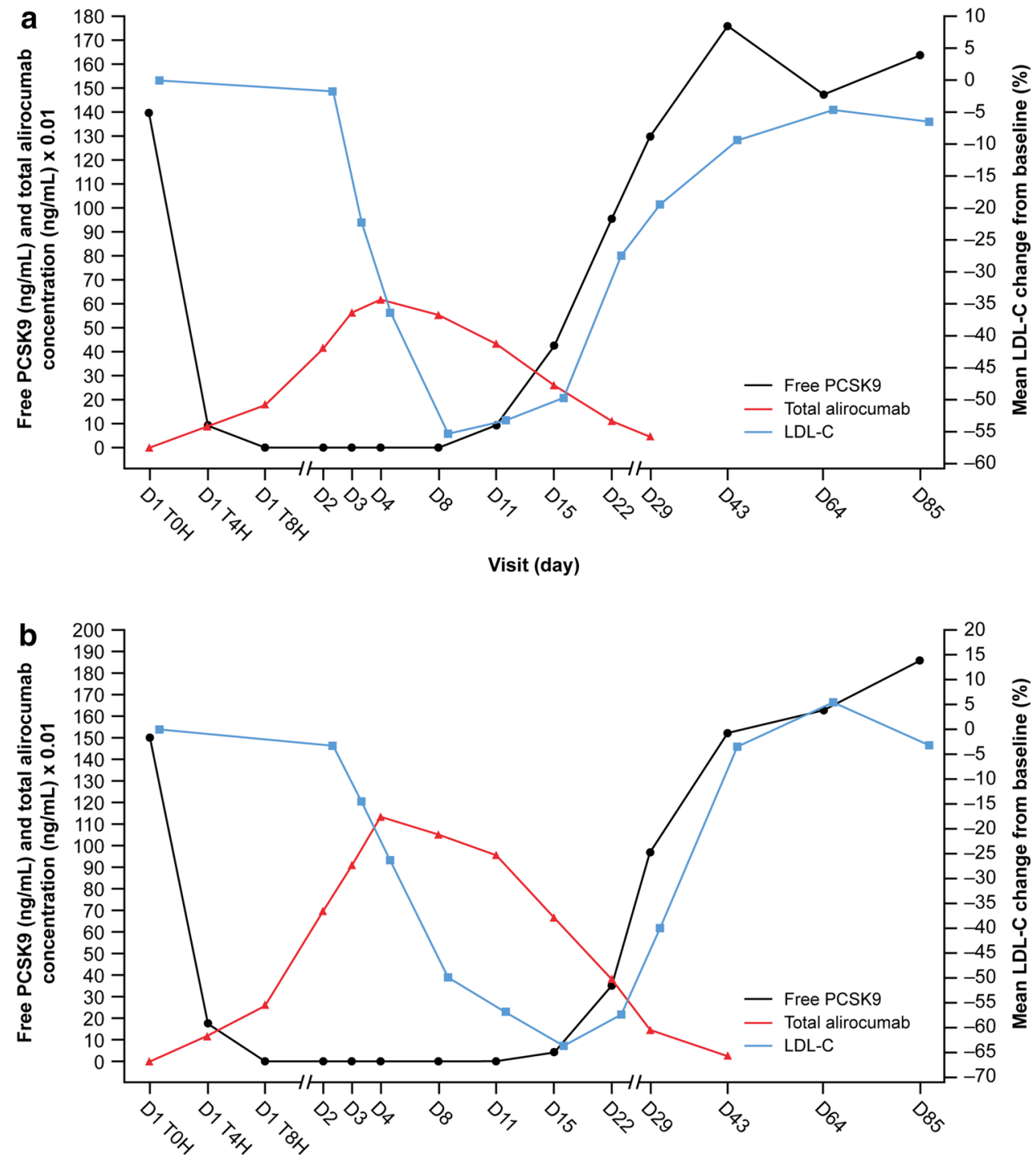

Visit (day)

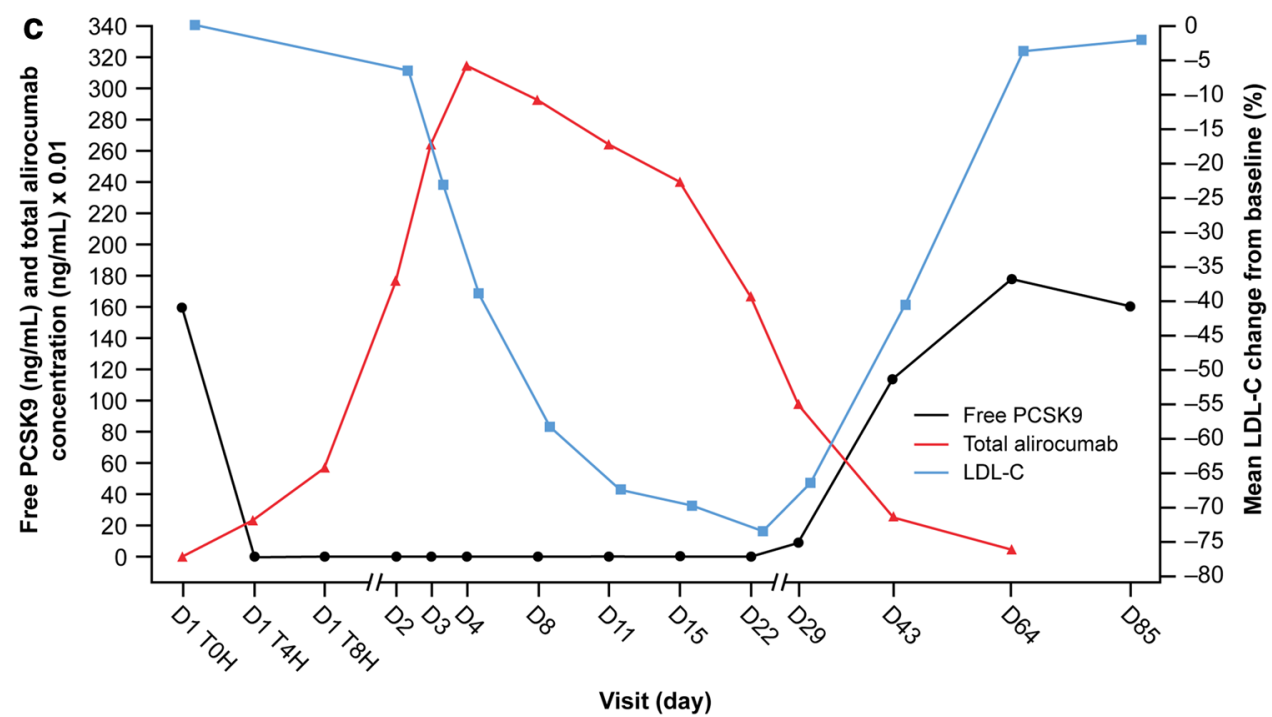


Fig. 6 Mean change (\%) from baseline (day 1) to EOS in a TC, b non-HDL-C, and $\mathbf{c}$ ApoB after subcutaneous singledose alirocumab or placebo administration. Baseline $=$ day 1 pre-dose assessment. Apo apolipoprotein, EOS end of study, non-HDL-C non-highdensity lipoprotein cholesterol, $S D$ standard deviation, $T C$ total cholesterol
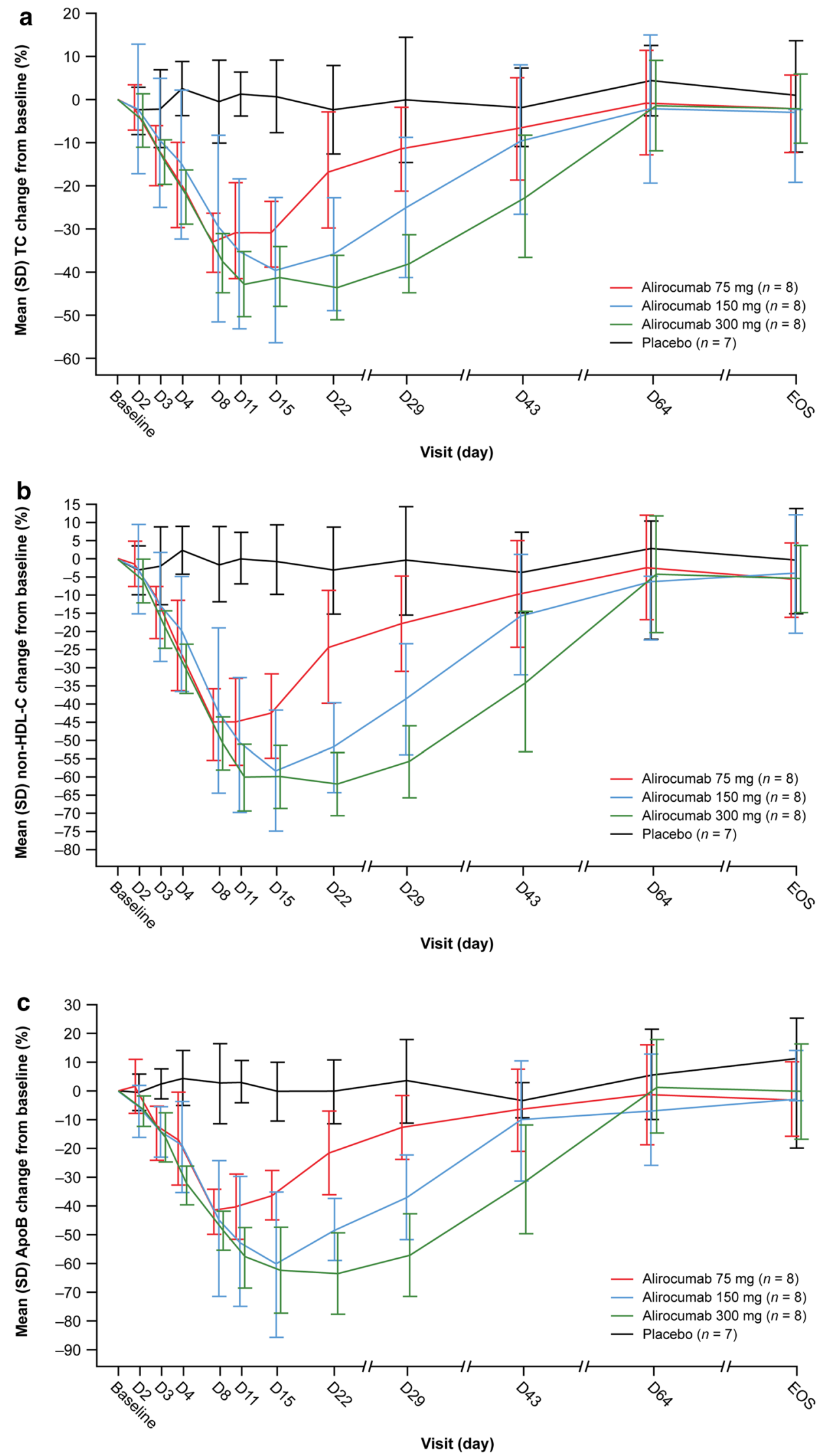
or $300 \mathrm{mg}$ ) were generally well-tolerated, and the pharmacokinetics and pharmacodynamics shown in this population, including effects on PCSK9 and LDL-C, were generally consistent with those reported in studies in the overall predominantly non-Asian population of the alirocumab clinical trial program [22, 25-28] and in phase I studies in healthy subjects of mixed ethnicity [29] and healthy Japanese men [30].

Pooled safety data from 14 phase II and III randomized trials showed a higher incidence of injection-site reactions in subjects receiving alirocumab than in controls [31]. In this study, on the day after alirocumab administration, a local injection-site reaction of mild intensity was reported for one recipient of alirocumab $300 \mathrm{mg}$, which spontaneously resolved within 2 days. Very low rates of immunogenicity have been reported in clinical trials of alirocumab. An immunogenicity analysis of patient data $(n=4747)$ pooled from ten phase III alirocumab trials found no association between the occurrence of ADAs (5.1\%) and the efficacy and safety of alirocumab, although injection-site reactions (mostly mild) were more frequent among patients with treatment-emergent ADAs than those without (11.6 vs. 5.9\%) [23]. All subjects who received alirocumab in the current study were ADA negative.

Consistent with the pharmacokinetics of alirocumab 75-300 mg in other populations [13, 22, 29], after single subcutaneous doses of alirocumab 75,150 , or $300 \mathrm{mg}$ in healthy Chinese subjects, alirocumab $C_{\max }$ occurred at a median of 3-7 days with generally low-to-moderate subject variability. Additionally, following single-dose subcutaneous administration of alirocumab $75 \mathrm{mg}$ in this healthy cohort, $C_{\text {max }}, t_{\max }, \mathrm{AUC}_{\infty}$, and $V_{\mathrm{ss}} / F$ were $6.32 \mathrm{mg} / \mathrm{L}, 3.00$ days, $87.6 \mathrm{mg} \cdot$ day $/ \mathrm{L}$, and $9.78 \mathrm{~L}$, respectively, similar to the data previously reported for a healthy cohort comprising various ethnicities $(8.18 \mathrm{mg} / \mathrm{L}, 2.96$ days, $129 \mathrm{mg} \cdot$ day/L, and 7.28 L, respectively) [29].

After subcutaneous administration of single-dose alirocumab 75, 150, and $300 \mathrm{mg}$, LDL-C levels were reduced from baseline from as early as day 3 , with maximum mean reductions on days 8 (55.3\%), 15 (63.7\%), and 22 (73.7\%), respectively. These percentage reductions are consistent with those reported in a pooled analysis of phase III alirocumab trials in patients with hypercholesterolemia receiving maximally tolerated background statin therapy in which, at week 12, LDL-C was reduced by $44.5-49.2 \%$ with alirocumab $75 \mathrm{mg} \mathrm{Q} 2 \mathrm{~W}$ and by $62.6 \%$ with alirocumab $150 \mathrm{mg}$ Q2W [32]. Additionally, the reductions were similar to those in data reported in a phase III study that investigated alirocumab $300 \mathrm{mg}$ every 4 weeks on maximally tolerated background statin therapy: at week 12, LDL-C was reduced by $55.3 \%$ [21]. In most clinical laboratories, LDL-C is not directly measured but is instead estimated using the Friedewald equation [24], which has limitations, particularly when LDL-C levels are low and triglyceride levels are elevated
( $>400 \mathrm{mg} / \mathrm{dL}$ ); however, this was not the case in our study. As shown in previous phase I trials [25], the magnitude and duration of LDL-C reduction were positively associated with the alirocumab dose administered and showed an inverse association with the observed increase in total PCSK9 levels, which reached a plateau from days 3 to 7 that persisted up to day $28(300 \mathrm{mg})$. Consistent with the target-mediated clearance of monoclonal antibodies [33], by binding to PCSK9, the apparent $\mathrm{CL} / F$ of alirocumab was reduced at the highest dose administered.

It has previously been shown that alirocumab has two elimination phases [34]: at low concentrations, elimination is predominately through saturable binding to PCSK9, whereas at higher concentrations, elimination is predominantly through a linear non-saturable proteolytic pathway [13]. At higher alirocumab doses, when alirocumab has bound all free PCSK9 and there is excess antibody (target saturation) [27], most of the total PSCK9 is in the biologically inert bound complex. With the elimination of the PCSK9-alirocumab complex being slow relative to formation, the concentration of total PCSK9 plateaus, indicating target saturation. Generally, the magnitude and duration of reduction in LDL-C is positively related to the alirocumab dose administered.

A population pharmacokinetic analysis of 2799 healthy volunteers or patients with hypercholesterolemia who received intravenous or subcutaneous alirocumab showed that age, bodyweight, creatinine clearance, sex, and race had no significant influence on the pharmacokinetic parameters of alirocumab; therefore, no dose adjustments are recommended for these populations [13, 27]. Furthermore, no meaningful difference in exposure was found between healthy Japanese and Caucasian subjects following single-dose subcutaneous administration of alirocumab 100-300 mg [35]. A recent study that qualified a population pharmacokinetic model for alirocumab in the Chinese population, including data from the present Chinese cohort, showed that alirocumab exposure parameters $\left(C_{\max }\right.$ and $\left.\mathrm{AUC}_{0-\mathrm{D} 14}\right)$ were similar to those reported in Caucasian/ Black populations and non-Chinese Asian patients [36]. The population pharmacokinetic modeling further supports the notion that the same alirocumab doses used globally are applicable to the Chinese population.

Administration of alirocumab led to reductions in LDL-C and in other atherogenic lipids/lipoproteins (TC, non-HDL$\mathrm{C}$, and $\mathrm{ApoB}$ ), peaking between days 8 and 22. Although no significant changes in HDL-C, triglycerides, VLDLC, ApoA1, or $L p(a)$ levels were seen in this study, trends for increases in HDL-C and ApoA1 and decreases in triglycerides, VLDL-C, and Lp(a) were noted. Notably, alirocumab has been shown to reduce $L p(a)$ levels $[25,37,38]$ : in a pooled analysis of ten alirocumab phase III trials, alirocumab 75/150 mg Q2W significantly reduced Lp(a) levels 
from baseline to week 24 by up to $29 \%$ [38]. Alirocumab was administered as a single dose in this study, which may explain the minor effect on levels of $\mathrm{Lp}$ (a) and other lipids/ lipoproteins.

The present study has several limitations. The upper age limit for study inclusion (45 years) precluded recruitment of older adults, and fewer female $(n=8$ [25.8\%]) than male $(n=23[74.2 \%])$ subjects were enrolled. Additionally, only healthy subjects, rather than those with prior ASCVD, were assessed; hence, studies in patients with ASCVD in China are required to evaluate the effects of alirocumab in this patient population. Results from the completed ODYSSEY EAST study, which investigated the efficacy and safety of alirocumab versus ezetimibe on background statin therapy in patients with hypercholesterolemia at high cardiovascular risk $(n=615)$ in China, India, and Thailand, showed that, consistent with previous ODYSSEY studies, alirocumab significantly reduced LDL-C versus ezetimibe in patients at high cardiovascular risk from Asia and was generally welltolerated [39].

\section{Conclusions}

Single-dose subcutaneous administration of alirocumab 75,150 , or $300 \mathrm{mg}$ was generally safe and well-tolerated in healthy Chinese subjects. The pharmacokinetic and pharmacodynamic parameters of alirocumab, including clinically meaningful reductions in LDL-C and other lipids/ lipoproteins, are consistent with those in data from Japanese [30] and Western populations [25, 29], suggesting that the alirocumab dosages used worldwide are applicable in the Chinese population.

Acknowledgements The authors thank the subjects, their families, and all investigators involved in the study at Peking University Third Hospital. Editorial comments on the manuscript were provided by Marc Israel, PharmD, BCPS, FCCP (Regeneron Pharmaceuticals, Inc.) and Aurélie Brunet, PharmD (Sanofi, Montpellier, France). Medical writing support under the direction of the authors was provided by Nila Bhana, MSc (Hons), of Prime (Knutsford, UK), supported by Sanofi and Regeneron Pharmaceuticals, Inc., according to good publication practice guidelines (http://annals.org/aim/article/2424869/good-publi cation-practice-communicating-company-sponsored-medical-resea rch-gpp3).

Author Contributions HL, YW, ZY, SZ, and XX contributed to data acquisition, data analysis and interpretation, and revising the manuscript critically for important intellectual content. MS, OV, YW, MTB$\mathrm{D}, \mathrm{YZ}$, and JL were involved in the study design; collection, analysis, and interpretation of data; revising the manuscript critically for important intellectual content; and data checking of information provided in the manuscript. All authors provided critical review of drafts and approved the final version for submission.

\section{Compliance with Ethical Standards}

Funding This study was funded by Sanofi and Regeneron Pharmaceuticals, Inc. Open access publication of this manuscript was sponsored by Sanofi and Regeneron Pharmaceuticals, Inc. The authors received no honoraria related to the development of this publication.

Conflict of interest HL, YW, ZY, SZ, and XX are employees of the Peking University Third Hospital. MS, OV, YWW, MTB-D and JL are employees of and shareholders in Sanofi. YZ is an employee of and shareholder in Regeneron Pharmaceuticals, Inc.

Ethical approval All procedures performed in the study participants were in accordance with the ethical standards of the independent ethics committee and with the 1964 Helsinki declaration and its later amendments.

Informed consent All participants provided written informed consent. No identifying information for individual subjects is included in this article.

Open Access This article is licensed under a Creative Commons Attribution-NonCommercial 4.0 International License, which permits any non-commercial use, sharing, adaptation, distribution and reproduction in any medium or format, as long as you give appropriate credit to the original author(s) and the source, provide a link to the Creative Commons licence, and indicate if changes were made. The images or other third party material in this article are included in the article's Creative Commons licence, unless indicated otherwise in a credit line to the material. If material is not included in the article's Creative Commons licence and your intended use is not permitted by statutory regulation or exceeds the permitted use, you will need to obtain permission directly from the copyright holder.To view a copy of this licence, visit http://creativecommons.org/licenses/by-nc/4.0/.

\section{References}

1. Mendis S, Puska P, Norving B, editors. Global Atlas on cardiovascular disease prevention and control. Geneva: World Health Organization; 2011.

2. Li H, Ge J. Cardiovascular diseases in China: current status and future perspectives. Int J Cardiol Heart Vasc. 2015;6:25-31.

3. Joint Committee for Guideline Revision. 2016 Chinese guidelines for the management of dyslipidemia in adults. J Geriatr Cardiol. 2018;15(1):1-29.

4. Zhao WH, Zhang J, Zhai Y, You Y, Man QQ, Wang CR, et al. Blood lipid profile and prevalence of dyslipidemia in Chinese adults. Biomed Environ Sci. 2007;20(4):329-35.

5. Zhao S, Wang Y, Mu Y, Yu B, Ye P, Yan X, et al. Prevalence of dyslipidaemia in patients treated with lipid-lowering agents in China: results of the DYSlipidemia International Study (DYSIS). Atherosclerosis. 2014;235(2):463-9.

6. Jiang J, Zhou YJ, Li JJ, Ge JB, Feng YQ, Huo Y. Uncontrolled hyperlipidemia in Chinese patients who experienced acute coronary syndrome: an observational study. Ther Clin Risk Manag. 2018;14:2255-64.

7. Zheng W, Zhang YJ, Bu XT, Guo XZ, Hu DY, Li ZQ, et al. LDL-cholesterol goal attainment under persistent lipid-lowering therapy in northeast China: Subgroup analysis of the dyslipidemia international study of China (DYSIS-China). Medicine. 2017;96(46):e8555. 
8. Catapano AL, Graham I, De Backer G, Wiklund O, Chapman MJ, Drexel H, et al. $2016 \mathrm{ESC} / \mathrm{EAS}$ guidelines for the management of dyslipidaemias. Eur Heart J. 2016;37(39):2999-3058.

9. Grundy SM, Stone NJ, Bailey AL, Beam C, Birtcher KK, Blumenthal RS, et al. 2018 AHA/ACC/AACVPR/AAPA/ABC/ACPM/ ADA/AGS/APhA/ASPC/NLA/PCNA Guideline on the Management of Blood Cholesterol: Executive Summary: a Report of the American College of Cardiology/American Heart Association Task Force on Clinical Practice Guidelines. J Am Coll Cardiol. 2019;73(24):3168-209.

10. Seidah NG, Benjannet S, Wickham L, Marcinkiewicz J, Jasmin $\mathrm{SB}$, Stifani S, et al. The secretory proprotein convertase neural apoptosis-regulated convertase 1 (NARC-1): liver regeneration and neuronal differentiation. Proc Natl Acad Sci USA. 2003;100(3):928-33.

11. Zhao Z, Tuakli-Wosornu Y, Lagace TA, Kinch L, Grishin NV, Horton JD, et al. Molecular characterization of loss-of-function mutations in PCSK9 and identification of a compound heterozygote. Am J Hum Genet. 2006;79(3):514-23.

12. Ference BA, Ginsberg HN, Graham I, Ray KK, Packard CJ, Bruckert E, et al. Low-density lipoproteins cause atherosclerotic cardiovascular disease. 1. Evidence from genetic, epidemiologic, and clinical studies. A consensus statement from the European Atherosclerosis Society Consensus Panel. Eur Heart J. 2017;38(32):2459-72.

13. Regeneron Pharmaceuticals Inc., sanofi-aventis US LLC. Praluent prescribing information. 2015. Available from: http://products. sanofi.us/praluent/praluent.pdf. Accessed 30 Apr 2019.

14. Sanofi. Praluent Prescribing Information. 2015. Available from: http://www.accessdata.fda.gov/drugsatfda_docs/label/2015/12555 9Orig 1 s000lbledt.pdf. Accessed 19 July 2016.

15. Amgen Inc. REPATHA Prescribing Information. 2015. Available from: https://www.pi.amgen.com/ /media/amgen/repositorysites/ pi-amgen-com/repatha/repatha_pi_hcp_english.pdf. Accessed 11 Mar 2019.

16. Amgen Inc. Amgen receives NMPA approval for Repatha ${ }^{\circledR}$ (evolocumab) in china to reduce the risk of cardiovascular events. 2019. Available from: https://wwwext.amgen.com/media/newsreleases/2019/01/amgen-receives-nmpa-approval-for-repathaevolocumab-in-china-to-reduce-the-risk-of-cardiovascular-event s/. Accessed 8 Mar 2019.

17. Robinson JG, Farnier M, Krempf M, Bergeron J, Luc G, Averna $\mathrm{M}$, et al. Efficacy and safety of alirocumab in reducing lipids and cardiovascular events. N Engl J Med. 2015;372(16):1489-99.

18. Schwartz GG, Steg PG, Szarek M, Bhatt DL, Bittner VA, Diaz R, et al. Alirocumab and cardiovascular outcomes after acute coronary syndrome. N Engl J Med. 2018;379(22):2097-107.

19. McKenney JM, Koren MJ, Kereiakes DJ, Hanotin C, Ferrand AC, Stein EA. Safety and efficacy of a monoclonal antibody to proprotein convertase subtilisin/kexin type 9 serine protease, SAR236553/REGN727, in patients with primary hypercholesterolemia receiving ongoing stable atorvastatin therapy. J Am Coll Cardiol. 2012;59(25):2344-53.

20. Stein EA, Gipe D, Bergeron J, Gaudet D, Weiss R, Dufour R, et al. Effect of a monoclonal antibody to PCSK9, REGN727/ SAR236553, to reduce low-density lipoprotein cholesterol in patients with heterozygous familial hypercholesterolaemia on stable statin dose with or without ezetimibe therapy: a phase 2 randomised controlled trial. Lancet. 2012;380(9836):29-36.

21. Roth EM, Moriarty PM, Bergeron J, Langslet G, Manvelian G, Zhao J, et al. A phase III randomized trial evaluating alirocumab $300 \mathrm{mg}$ every 4 weeks as monotherapy or add-on to statin: ODYSSEY CHOICE I. Atherosclerosis. 2016;254:254-62.

22. Rey J, Poitiers F, Paehler T, Brunet A, DiCioccio AT, Cannon CP, et al. Relationship between low-density lipoprotein cholesterol, free proprotein convertase subtilisin/kexin type 9 , and alirocumab levels after different lipid-lowering strategies. J Am Heart Assoc. 2016;5(6): 0003323

23. Roth EM, Goldberg AC, Catapano AL, Torri A, Yancopoulos GD, Stahl N, et al. Antidrug antibodies in patients treated with alirocumab. N Engl J Med. 2017;376(16):1589-90.

24. Friedewald WT, Levy RI, Fredrickson DS. Estimation of the concentration of low-density lipoprotein cholesterol in plasma, without use of the preparative ultracentrifuge. Clin Chem. 1972;18(6):499-502.

25. Stein EA, Mellis S, Yancopoulos GD, Stahl N, Logan D, Smith WB, et al. Effect of a monoclonal antibody to PCSK9 on LDL cholesterol. N Engl J Med. 2012;366(12):1108-18.

26. Roth EM, McKenney JM, Hanotin C, Asset G, Stein EA. Atorvastatin with or without an antibody to PCSK9 in primary hypercholesterolemia. N Engl J Med. 2012;367(20):1891-900.

27. Martinez JM, Brunet A, Hurbin F, DiCioccio AT, Rauch C, Fabre D. Population pharmacokinetic analysis of alirocumab in healthy volunteers or hypercholesterolemic subjects using a MichaelisMenten approximation of a target-mediated drug disposition model-support for a biologics license application submission: part I. Clin Pharmacokinet. 2019;58(1):101-13.

28. Nicolas X, Djebli N, Rauch C, Brunet A, Hurbin F, Martinez JM, et al. Population pharmacokinetic/pharmacodynamic analysis of alirocumab in healthy volunteers or hypercholesterolemic subjects using an indirect response model to predict low-density lipoprotein cholesterol lowering: support for a biologics license application submission: part II. Clin Pharmacokinet. 2019;58(1):115-30.

29. Lunven C, Paehler T, Poitiers F, Brunet A, Rey J, Hanotin C, et al. A randomized study of the relative pharmacokinetics, pharmacodynamics, and safety of alirocumab, a fully human monoclonal antibody to PCSK9, after single subcutaneous administration at three different injection sites in healthy subjects. Cardiovasc Ther. 2014;32(6):297-301.

30. Teramoto T, Kobayashi M, Uno K, Takagi Y, Matsuoka O, Sugimoto M, et al. Efficacy and safety of alirocumab in Japanese subjects (Phase 1 and 2 studies). Am J Cardiol. 2016;118(1):56-63.

31. Jones PH, Bays HE, Chaudhari U, Pordy R, Lorenzato C, Miller $\mathrm{K}$, et al. Safety of alirocumab (a PCSK9 monoclonal antibody) from 14 randomized trials. Am J Cardiol. 2016;118(12):1805-11.

32. Farnier M, Gaudet D, Valcheva V, Minini P, Miller K, Cariou B. Efficacy of alirocumab in high cardiovascular risk populations with or without heterozygous familial hypercholesterolemia: pooled analysis of eight ODYSSEY Phase 3 clinical program trials. Int J Cardiol. 2016;223:750-7.

33. Chaparro-Riggers J, Liang H, DeVay RM, Bai L, Sutton JE, Chen $\mathrm{W}$, et al. Increasing serum half-life and extending cholesterol lowering in vivo by engineering antibody with $\mathrm{pH}$-sensitive binding to PCSK9. J Biol Chem. 2012;287(14):11090-7.

34. Djebli N, Martinez JM, Lohan L, Khier S, Brunet A, Hurbin F, et al. Target-mediated drug disposition population pharmacokinetics model of alirocumab in healthy volunteers and patients: pooled analysis of randomized phase I/II/III studies. Clin Pharmacokinet. 2017;56(10):1155-71.

35. European Medicines Agency. European Public Assessment Report (EPAR) WC500194524. 2015. Available from: http://www.ema. europa.eu/docs/en_GB/document_library/EPAR_-_Public_asses sment_report/human/003882/WC500194524.pdf. Accessed 30 Apr 2019.

36. Calvier EAM, Martinez JM, Fabre D, Brunet A, Zhang YY. Alirocumab population pharmacokinetics in Chinese patients using priors. In: Annual meeting population approach group in Europe (PAGE), Stockholm. 2019. p. Abstr 8838.

37. Gaudet D, Kereiakes DJ, McKenney JM, Roth EM, Hanotin C, Gipe D, et al. Effect of alirocumab, a monoclonal proprotein convertase subtilisin/kexin 9 antibody, on lipoprotein(a) 
concentrations (a pooled analysis of $150 \mathrm{mg}$ every two weeks dosing from phase 2 trials). Am J Cardiol. 2014;114(5):711-5.

38. Gaudet D, Watts GF, Robinson JG, Minini P, Sasiela WJ, Edelberg J, et al. Effect of alirocumab on lipoprotein(a) over $\geq 1.5$ years (from the Phase 3 ODYSSEY program). Am J Cardiol. 2017;119(1):40-6
39. Han Y, Chen J, Chopra V, Zhang S, Su G, Ma C, et al. ODYSSEY EAST: alirocumab efficacy and safety vs ezetimibe in high cardiovascular risk patients with hypercholesterolemia and on maximally tolerated statin in China, India, and Thailand. J Clin Lipidol. 2019. https://doi.org/10.1016/j.jacl.2019.10.015.

\section{Affiliations}

\section{Haiyan $\mathrm{Li}^{1,2}$-Yudong $\mathrm{Wei}^{2} \cdot$ Zhenhua Yang $^{2}$. Shuang Zhang ${ }^{2} \cdot$ Xiuxiu Xu $^{2} \cdot$ Mengmeng Shuai $^{3} \cdot$ Olivier Vitse ${ }^{4}$. Yiwen $\mathrm{Wu}^{3} \cdot$ Marie T. Baccara-Dinet ${ }^{4} \cdot$ Yi Z Zhang $^{5} \cdot$ Jianyong $\mathrm{Li}^{6}$}

1 Department of Cardiology, NHC Key Laboratory of Cardiovascular Molecular Biology and Regulatory Peptides, Peking University Third Hospital, 49 North Garden Road, Haidian Distrct, Beijing 100191, China

2 Drug Clinical Trial Center, Peking University Third Hospital, 49 North Garden Road, Haidian Distrct, Beijing 100191, China

3 Sanofi, Beijing, China
4 Clinical Development R\&D, Sanofi, Montpellier, France

5 Regeneron Pharmaceuticals, Inc., Tarrytown, NY, USA

6 Sanofi R\&D, Shanghai, China 